\title{
A GENERALIZATION OF MINIMAL CONES
}

\author{
BY
}

NORIO EJIRI

\begin{abstract}
Let $R_{+}$be a positive real line, $S^{n}$ an $n$-dimensional unit sphere. We denote by $R_{+} \times S^{n}$ the polar coordinate of an $(n+1)$-dimensional Euclidean space $R^{n+1}$. It is well known that if $M$ is a minimal submanifold in $S^{n}$, then $R_{+} \times M$ is minimal in $R^{n+1} . R_{+} \times M$ is called a minimal cone. We generalize this fact and give many minimal submanifolds in real and complex space forms.
\end{abstract}

1. Introduction. The existence and global behavior of submanifold with constant mean curvature is, in full generality, a difficult area of study. The nonlinearity of the problem makes even the construction of explicit examples reasonably difficult and, at the same time, makes such examples indispensable guidelines for research.

Let $M$ be a Riemannian manifold and $G$ a compact connected group of isometries of $M$. Then W. Y. Hsiang and H. B. Lawson, Jr. [7] proved that a $G$-invariant submanifold $N$ of $M$ is minimal if and only if $N / G$ is minimal in $M / G$ with the appropriate metric. Under this observation, they explicitly construct vast numbers of compact minimal submanifolds in nearly all homogeneous spaces.

In [13], J. Simons gives a powerful method of analyzing compact minimal submanifolds in a sphere and a complex projective space. Nowadays it is known that there exist many pinching theorems.

It is natural that we are interested in the relaxation among those pinching's values. The purpose of this paper is to give two observations for the construction of complete submanifolds with constant mean curvature in a real and complex space form and to see the relation between examples obtained by our discussion and some pinching theorems.

Our two observations are as follows:

First observation. Let $B$ and $F$ be, respectively, a Riemannian manifold with metric $g_{1}$ and a Riemannian manifold with metric $g_{2}$. Let $f$ be a positive function on $B$. We denote by $B \times{ }_{f} F$ the product manifold $B \times F$ of $B$ and $F$ with metric $g=g_{1}+f^{2} g_{2} . B \times{ }_{f} F$ is called the warped product of $B \times F[1]$. Let $M$ and $N$ be an $m$-dimensional submanifold of $B$ and an $n$-dimensional submanifold of $F$, respectively. We may naturally see that $M \times N$ is an $(m+n)$-dimensional submanifold of $B \times{ }_{f} F$, and, with respect to the induced metric, it is also a warped product $M \times{ }_{f} N$.

Received by the editors November 3, 1981 and, in revised form, March 17, 1982.

1980 Mathematics Subject Classification. Primary 53C40; Secondary 53C25.

Key words and phrases. Minimal cone, cohomogeneity, catenoid, warped product, Sasakian structure, $\Phi$-holomorphic sectional curvature, totally real submanifold.

(C) 1983 American Mathematical Society 0002-9947/82/0000-0295/\$05.25 
The first observation is that

$M \times N$ is minimal in $B \times{ }_{f} F$ if and only if

(i) $M$ is minimal in $B$ with metric $f^{2 n / m} g_{1}$ and

(ii) $N$ is minimal in $F$.

If $B$ is a positive real line $R_{+}$whose coordinate function is $s, F$ is a $q$-dimensional unit sphere $S^{q}$ and $f=s$, then $R_{+} \times{ }_{s} S^{q}$ becomes a $(q+1)$-dimensional Euclidean space $R^{q+1}$ minus the origin $o$. If $M=R_{+}$and $N$ is a minimal submanifold in $S^{q}$, then so is $R_{+} \times N$. This is well known as a minimal cone. On the other hand, in case $N=F$ is a compact connected homogeneous Riemannian manifold $G / K, G$ acts naturally on $B \times{ }_{f} F$ as isometries. Thus $M \times{ }_{f} F$ is a $G$-invariant minimal submanifold with cohomogeneity $m$ if $M$ satisfies the condition (i).

Second observation. Let $M$ be a $(2 n-1)$-dimensional Sasakian manifold with Sasakian structure $(\phi, \xi, \eta, g)$ and $I_{1}$ an interval $(a, b)$ with coordinate function $s$. Let $f$ and $h$ be functions on $I_{1}$ such that $f>0$ and $f^{2}+h>0$. We define a metric $\tilde{g}=d s^{2}+f^{2} g+h \eta \otimes \eta$. For an $(n-1)$-dimensional integral submanifold $N$ of $M$ and a regular curve $r=(s(u), t(u))$ of an interval $I_{2}$ into $I_{1} \times R$, we construct an immersion $\chi$ of $I_{2} \times N$ into $I_{1} \times M$ given by $\chi(u, x)=\left(s(u), \operatorname{Exp}_{x} t(u) \xi\right)$. Then the second observation is given as follows:

$I_{2} \times N$ is minimal in $I_{1} \times M$ with metric $\tilde{g}$ if and only if

(iii) $r$ is a geodesic in $I_{1} \times R$ with metric

$$
f^{2(n-1)}\left\{d s^{2}+\left(f^{2}+h\right) d t^{2}\right\}
$$

and

(iv) $N$ is minimal in $M$.

From these two observations, we proceed by elementary technique to explicitly construct many complete minimal submanifolds in a real and complex space form.

The author expresses his deep gratitude to Professor K. Ogiue who encouraged him and gave him a lot of valuable suggestions.

2. Warped product. R. L. Bishop and B. O'Neill [1] studied some properties of warped product. We shall review them. Let $B$ and $F$ be, respectively, a $p$-dimensional Riemannian manifold with metric $g_{1}$ and a $q$-dimensional Riemannian manifold with metric $g_{2}$. Let $f$ be a positive function on $B$. We denote by $B \times{ }_{f} F$ the product manifold $B \times F$ of $B$ and $F$ with metric $g=g_{1}+f^{2} g_{2}$.

LEMMA 1 [1, p. 23]. $B \times_{f} F$ is complete if and only if $B$ and $F$ are complete.

Let $\nabla, \nabla_{1}$ and $\nabla_{2}$ be the covariant differentiations for $g, g_{1}$ and $g_{2}$ respectively.

LeMma 2 [1, p. 24]. Let $X, Y$ be vector fields on $B$, and $V, W$ vector fields on $F$. Then

(1) $\nabla_{X} Y=\nabla_{1 X} Y$,

(2) $\nabla_{X} V=\nabla_{V} X=(X f / f) V$,

(3) $\nabla_{V} W=-(g(V, W) / f) G+\nabla_{2 V} W$,

where $G$ is the gradient of $f$.

We denote by $R, R_{1}$ and $R_{2}$ the curvature tensors of $B \times{ }_{f} F, B$ and $F$ respectively. 
Lemma 3 [1, p. 25]. Let $X, Y, Z$ be vector fields on $B$, and $U, V, W$ vector fields on $F$. Then

(1) $R_{U V} W=R_{2 U V} W-(\|G\| / f)^{2}[g(U, W) V-g(V, W) U]$,

(2) $R_{X V} Y=-\left(g\left(\nabla_{X} G, Y\right) / f\right) V$,

(3) $R_{X Y} U=R_{V W} X=0$,

(4) $R_{X V} W=R_{X W} V=(g(V, W) / f) \nabla_{X} G$,

(5) $R_{X Y} Z=R_{1 X Y} Z$.

It follows from Lemma 3 that

LEMMA 4. $B \times_{f} F$ has constant sectional curvature $c$ if and only if

(1) the sectional curvature of $B$ is $c$ and that of $F$ is $c f^{2}+\|G\|^{2}$,

(2) Hess $f=-c f g_{1}$.

REMARK. (2) implies that $c f^{2}+\|G\|^{2}$ is constant.

We denote by $S^{q}, R^{q}$ and $H^{q}$ a $q$-dimensional unit sphere, Euclidean space and hyperbolic space of sectional curvature -1 , respectively.

COROllary 1. (1) $\left[(0, \pi / 2) \times_{\sin s} S^{p-1}\right] \times_{\cos s} S^{q}$ is isometric to an open set of $S^{p+q}$

(2) $\left(R_{+} \times R^{p-1}\right) \times{ }_{s} S^{q}$ is isometric to an open set of $R^{p+q}$,

(3) $\left(R \times \times_{e^{s}} R^{p-1}\right) \times_{e^{s}} R^{q}=H^{p+q}$,

(4) $\left(R \times{ }_{\sinh s} H^{p-1}\right) \times_{\cosh s} H^{q}$ is isometric to an open set of $H^{p+q}$,

(5) $\left(R_{+} \times_{\cosh s} H^{p-1}\right) \times_{\sinh s} S^{q}$ is isometric to an open set of $H^{p+q}$.

3. Geodesic coordinates of complex space forms. Let $M$ be a $(2 n-1)$-dimensional Sasakian manifold with Sasakian structure $(\phi, \xi, \eta, g)$ (cf., for example, [14]).

$$
\begin{gathered}
\phi \xi=0, \quad \eta(\xi)=1, \quad \phi^{2}=-I+\xi \otimes \eta, \\
g(X, \xi)=\eta(X), \quad g(\phi X, \phi Y)=g(X, Y)-\eta(X) \eta(Y), \\
d \eta(X, Y)=2 g(\phi X, Y), \\
\phi X=\nabla_{X} \xi, \quad\left(\nabla_{X} \phi\right) Y=\eta(Y) X-g(X, Y) \xi,
\end{gathered}
$$

where $\nabla$ is the covariant differentiation for $g$.

Denote by $K(X, Y)$ the sectional curvature for the 2-plane spanned by $X$ and $Y$. $M$ is said to have constant $\phi$-holomorphic sectional curvature if $K(X, \phi X)$ is constant for any $X \neq 0$ such that $\eta(X)=0$ [11]. A Sasakian manifold $M$ has constant $\phi$-holomorphic sectional curvature $\rho$ if and only if the curvature tensor $R$ satisfies

$$
\begin{aligned}
R_{X Y} Z= & ((\rho+3) / 4)(g(X, Z) Y-g(Y, Z) X)+((\rho-1) / 4) \\
\cdot[ & \eta(X) g(Y, Z)+\eta(Y) \eta(Z) X-\eta(Y) g(X, Z) \xi \\
& -\eta(X) \eta(Z) Y+g(\phi X, Z) \phi Z-g(\phi Y, Z) \phi X+2 g(\phi X, Y) \phi Z] .
\end{aligned}
$$

S. Tanno gives the model spaces $S^{2 n-1}[\rho](\rho>-3), E^{2 n-1}[-3]$ and $\left(L \cdot C D^{n-1}\right)[\rho]$ $(\rho<-3)$ of constant $\phi$-holomorphic sectional curvature $\rho$ and proves the uniqueness of them. That is, we see 
Proposition 2 [14]. Let $M$ be a $(2 n-1)$-dimensional complete simply connected Sasakian manifold of constant $\phi$-holomorphic sectional curvature.

(1) If $\rho>-3, M$ is isomorphic to $S^{2 n-1}[\rho]$.

(2) If $\rho=-3, M$ is isomorphic to $E^{2 n-1}[-3]$.

(3) If $\rho<-3, M$ is isomorphic to $\left(L \cdot C D^{n-1}\right)[\rho]$.

REMARK. $S^{2 n-1}[1]$ is $S^{2 n-1}$.

For the second time we denote by $M$ a $(2 n-1)$-dimensional Sasakian manifold with Sasakian structure $(\phi, \xi, \eta, g)$. Let $I$ be an open interval $(a, b)$ and $s$ a coordinate function on $I$. Let $f$ and $h$ be functions in $I$ such that $f>0$ and $f^{2}+h>0$. Then $\tilde{g}$ defined by $d s^{2}+f^{2} g+h \eta \otimes \eta$ is a metric on $I \times M$. We denote by $\tilde{\nabla}$ the covariant differentiation on $I \times M$ for $\tilde{g}$.

LeMma 5. Let $X$ and $Y$ be vector fields on $M$ such that $\eta(X)=\eta(Y)=0$. Then

$$
\begin{gathered}
\tilde{\nabla}_{\partial / \partial s^{\partial}} \partial / \partial s=0, \quad \tilde{\nabla}_{\partial / \partial s} X=\tilde{\nabla}_{X} \partial / \partial s=\left(f^{\prime} / f\right) X, \\
\tilde{\nabla}_{\partial / \partial s} \xi=\tilde{\nabla}_{\xi} \partial / \partial s=\left(f^{2}+h\right)^{\prime} / 2\left(f^{2}+h\right) \xi, \\
\tilde{\nabla}_{X} Y=-\left(f^{\prime} / f\right) \tilde{g}(X, Y) \partial / \partial s+\nabla_{X} Y, \quad \tilde{\nabla}_{X} \xi=\left(f^{2}+h\right) / f^{2} \phi X, \\
\tilde{\nabla}_{\xi} X=\nabla_{\xi} X+\left(h / f^{2}\right) \phi X, \quad \tilde{\nabla}_{\xi} \xi=-\left(f^{2}+h\right)^{\prime} / 2 \partial / \partial s,
\end{gathered}
$$

where $f^{\prime}=\partial f / \partial s$ etc.

It is well known that there is a complex structure on $R \times M$. We construct an almost complex structure $J$ for which $\tilde{g}$ is an almost Hermitian metric:

$$
\begin{gathered}
J \partial / \partial s=\left(1 / \sqrt{f^{2}+h}\right) \xi, \quad J \xi=-\sqrt{f^{2}+h} \partial / \partial s, \\
J X=\phi X \text { for any } X \text { such that } \eta(X)=0 .
\end{gathered}
$$

LeMMA 6. $\tilde{g}$ is a Kählerian metric with respect to $J$ if and only if $h=f^{2}\left(f^{\prime 2}-1\right)$.

Let $\tilde{R}$ be the curvature tensor for the Kählerian metric. By a simple but long calculation, we obtain

LEMMA 7. Let $X, Y$ and $Z$ be vector fields on $M$ such that $\eta(X)=\eta(Y)=\eta(Z)=0$. Then

$$
\begin{gathered}
\tilde{R}_{\partial / \partial s X} \partial / \partial s=-\left(f^{\prime \prime} / f\right) X, \quad \tilde{R}_{\partial / \partial s X} Y=f f^{\prime \prime} g(X, Y) \partial / \partial s+\left(f^{\prime \prime} / f^{\prime}\right) g(\phi X, Y) \xi, \\
\tilde{R}_{\partial / \partial s X} \xi=-f^{\prime} f^{\prime \prime} \phi X, \quad \tilde{R}_{\partial / \partial s \xi} \partial / \partial s=-\left(3 f f^{\prime 2} f^{\prime \prime}+f^{2} f^{\prime} f^{\prime \prime \prime}\right) / f^{2} f^{\prime 2} \xi, \\
\tilde{R}_{\partial / \partial s \xi} X=-2 f^{\prime} f^{\prime \prime} \phi X, \quad \tilde{R}_{\partial / \partial s \xi} \xi=\left(3 f f^{\prime 2}+f^{2} f^{\prime} f^{\prime \prime \prime}\right) \partial / \partial s, \\
\tilde{R}_{X Y} \partial / \partial s=-\left(2 f^{\prime \prime} / f^{\prime}\right) g(\phi X, Y) \xi, \\
\tilde{R}_{X Y} Z=-2 g(\phi X, Y)\left(f^{\prime 2}-1\right) \phi Z+f^{\prime 2} g(Y, Z) X-f^{\prime 2} g(X, Z) Y \\
+\left(1-f^{\prime 2}\right) g(\phi Z, Y) \phi X-\left(1-f^{\prime 2}\right) g(\phi Z, X) \phi Y+R_{X Y} Z, \\
\tilde{R}_{X Y} \xi=2 f^{2} f^{\prime} f^{\prime \prime} g(\phi X, Y) \partial / \partial s, \quad \tilde{R}_{X \xi} \partial / \partial s=-f^{\prime} f^{\prime \prime} \phi X, \\
\tilde{R}_{X \xi} Y=-f^{2} f^{\prime} f^{\prime \prime} g(X, \phi Y) \partial / \partial s-\left(1+f f^{\prime \prime}\right) g(X, Y) \xi+R_{X \xi} Y, \\
\tilde{R}_{X \xi} \xi=f f^{\prime 2} f^{\prime \prime} X,
\end{gathered}
$$

where $R$ is the curvature tensor of $M$. 
Combining the above with (3.1), we have

LEMMA 8. The Kählerian manifold $(I \times M, J, \tilde{g})$ is of constant holomorphic sectional curvature $4 c$ if and only if

(1) $f^{\prime \prime}=-c f$ and

(2) $M$ has constant $\phi$-holomorphic sectional curvature $4\left(c f^{2}+f^{\prime 2}\right)-3$.

In particular, $\left((0, \pi / 2) \times S^{2 n-1}, J, \tilde{g}\right)$ with $f=\sin s$ is a geodesic coordinate neighborhood of an n-dimensional complex projective space $P_{n}$ of constant holomorphic sectional curvature 4 and $\left(R_{+} \times S^{2 n-1}, J, \tilde{g}\right)$ with $f=s$ and $\sinh s$ is respectively $a$ geodesic coordinate neighborhood of an n-dimensional complex Euclidean space $\mathbf{C}^{n}$ and an $n$-dimensional complex hyperbolic space $H_{n}$ of constant holomorphic sectional curvature -4 .

REMARK. $I \times M$ is an Einstein Kähler manifold with Ricci curvature $\lambda$ if and only if

(1) Ricci curvature of $M$ in the direction of $X$ such that $\eta(X)=0$ is a constant $\mu$ and

(2) $2 f f^{\prime \prime}+2 n f^{\prime 2}+\lambda f^{2}-(2+\mu)=0$.

4. First observation. We use the same notation and terminologies as in $\S 2$. Let $M$ be an $m$-dimensional submanifold in $B$ and $N$ an $n$-dimensional submanifold in $F$. We denote by $\sigma_{1}$ and $\sigma_{2}$ the second fundamental forms of $M$ and $N$ respectively. Hence we may assume that $M \times N$ is an $(m+n)$-dimensional submanifold in $B \times{ }_{f} F$. The second fundamental form of $M \times N$ is denoted by $\sigma$.

LEMMA 9. Let $X, Y$ be vector fields on $M$, and $V, W$ vector fields on $N$. Then

(1) $\sigma(X, Y)=\sigma_{1}(X, Y)$,

(2) $\sigma(X, V)=0$,

(3) $\sigma(V, W)=-f g_{2}(V, W) G^{\perp}+\sigma_{2}(V, W)$,

where $G^{\perp}$ is the normal component of $G$ for $M$ in $B$.

Accordingly, we get

LEMMA 10. The mean curvature vector of $M \times N$ in $B \times{ }_{f} F$ is given by

$$
m /(m+n) \mathcal{L}_{1}-n /((m+n) f) G^{\perp}+n /\left((m+n) f^{2}\right) \mathcal{L}_{2}
$$

where $\mathcal{L}_{1}$ and $\mathcal{L}_{2}$ are respectively the mean curvature vectors of $M$ and $N$.

On the other hand, under a conformal change of the metric of the ambiant space, the mean curvature vector of a submanifold is subject to some change.

LEMMA 11. Let $\mathcal{L}^{\prime}$ be the mean curvature vector of $M$ in $B$ with metric $f^{2 n / m} g_{1}$. Then

$$
\varrho^{\prime}=f^{-2 n / m}\left(\varrho_{1}-n /(m f) G^{\perp}\right) .
$$

Lemma 11, together with Lemma 10, shows the first observation.

THEOREM 1. $M \times N$ is minimal in $B \times{ }_{f} F$ if and only if

(1) $M$ is minimal in $B$ with metric $f^{2 n / m} g_{1}$ and

(2) $N$ is minimal in $F$. 
Applying Theorem 1 to Corollary 1 , we have some examples of minimal submanifolds in a real space form.

EXAMPLE 1. For (1) of Corollary 1 , let $N$ be an $n$-dimensional minimal submanifold in $S^{q}$ and $M$ an $m$-dimensional minimal submanifold in $(0, \pi / 2) \times S^{p-1}$ with metric $(\cos s)^{2 n / m}\left(d s^{2}+(\sin s)^{2} g\right)$. Then $M \times N$ can be minimally immersed in $S^{p+q}$.

EXAMPLE 2. For (2), let $N$ be an $n$-dimensional minimal submanifold in $S^{q}$ and $M$ an $m$-dimensional minimal submanifold in $R_{+} \times R^{p-1}$ with metric $s^{2 n / m}\left(d s^{2}+g\right)$. Then $M \times N$ can be minimally immersed in $R^{p+q}$.

EXAMPLE 3. For (3), let $N$ be an $n$-dimensional minimal submanifold in $R^{q}$ and $M$ an $m$-dimensional minimal submanifold in $R \times R^{p-1}$ with metric

$$
e^{(2 n / m) s}\left(d s^{2}+e^{2 s} g\right) \text {. }
$$

Then $M \times N$ can be minimally immersed in $H^{p+q}$.

EXAMPLE 4. For (4), let $N$ be an $n$-dimensional minimal submanifold in $H^{q}$ and $M$ an $n$-dimensional minimal submanifold of $R_{+} \times H^{p^{-1}}$ with metric

$$
(\cosh s)^{2 n / m}\left(d s^{2}+(\sinh s)^{2} g\right) \text {. }
$$

Then $M \times N$ can be minimally immersed in $H^{p+q}$.

In particular, we have simple cases in Examples 3 and 4.

EXAmple $3^{\prime}$. Let $N$ be a complete minimal submanifold in $R^{q}$. Then $R \times{ }_{e^{s}} N$ is a complete minimal submanifold in $H^{q+1}$.

EXAMPLE $4^{\prime}$. Let $N$ be a complete minimal submanifold in $H^{q}$. Then $R \times{ }_{\cosh s} N$ is a complete minimal submanifold in $H^{q+1}$.

EXAMPLE 5. For (5), let $N$ be an $n$-dimensional minimal submanifold in $S^{q}$ and $M$ an $m$-dimensional minimal submanifold in $R_{+} \times H^{p-1}$ with metric

$$
(\sinh s)^{2 n / m}\left(d s^{2}+(\cosh s)^{2} g\right) \text {. }
$$

Then $M \times N$ can be minimally immersed in $H^{p+q}$.

5. Complete submanifolds with parallel mean curvature vector in real space forms. If $p=2$ in each example of $\$ 4$, then $M$ is a geodesic of a surface with "some metric". More generally this section is devoted to the study of curves $M(=\gamma)$ for each case such that $\gamma \times N$ has constant mean curvature.

EXAMPLE 6. $\left[(0, \pi / 2) \times_{\sin s} S^{1}\right] \times_{\cos s} S^{q}$ is isometric to an open set of $S^{2+q}$. Let $N$ be an $n$-dimensional minimal submanifold and $\gamma$ a nonparametric curve $(s(t), t)$ in $(0, \pi / 2) \times_{\sin s} S^{1}$ whose unit normal vector field $\mathscr{N}$ is given by

$$
((\sin s) \partial / \partial s-\dot{s} /(\sin s) \partial / \partial t) / \sqrt{\dot{s}^{2}+\sin ^{2} s},
$$

where $\dot{s}=\partial s / \partial t$. It follows from Lemma 10 that the mean curvature vector of $\gamma \times N$ is $1 /(n+1) \mathscr{L}_{1}+(n /(n+1)) \tan s[\partial / \partial s]^{\perp}$, which has constant length $H$ if and only if the innerproduct of $\mathcal{N}$ and $\mathcal{L}$ is $H$ or $-H$. From Lemma 2, we get an ordinary differential equation for $s$ :

$$
\begin{aligned}
& \left\{(\ddot{s}-(\sin s)(\cos s))(\sin s)-2 \dot{s}^{2} \cos s\right\} /{\sqrt{\dot{s}^{2}+\sin ^{2} s}}^{3} \\
& +n(\sin s)(\tan s) / \sqrt{\dot{s}^{2}+\sin ^{2} s}= \pm(n+1) H,
\end{aligned}
$$


which leads to

$$
\left(\sin ^{2} s \cos ^{n} s / \sqrt{\dot{s}^{2}+\sin ^{2} s}\right)^{\bullet} \mp H\left(\cos ^{n+1} s\right)^{\bullet}=0 .
$$

Hence there is a constant $c$ such that

$$
\dot{s}^{2}=\sin ^{2} s\left\{\sin ^{2} s \cos ^{2 n} s /\left( \pm H \cos ^{n+1} s+c\right)^{2}-1\right\} .
$$

To simplify the analysis of solutions in (5.1), we consider the case where the right-hand side of $(5.1)$ is $(n+1) H$ and $c>0$. These guarantee $H \cos ^{n+1} s+c>0$. We may assume that $s$ satisfies the initial conditions

$$
s(0)=a, \quad \dot{s}(0)=0, \quad \ddot{s}(0)>0 .
$$

Then

$$
\begin{aligned}
& c=-H \cos ^{n+1} a+(\sin a) \cos ^{n} a \text { and } \\
& H<\tan a<\left\{((n+1) / n) H+\sqrt{((n+1) / n)^{2} H^{2}+4 / n}\right\} / 2 .
\end{aligned}
$$

It is easy to see that there is $b$ such that $0<a<b<\pi / 2$ and

$$
\cos ^{n+1} b(\tan b-H)=\cos ^{n+1} a(\tan a-H) .
$$

We denote by $\Omega_{a}$ the smallest positive $t$ such that $\dot{s}(t)=0$. Then $s$ is an increasing function on $\left[0, \Omega_{a}\right]$ and $s\left(\Omega_{a}\right)=b$. In fact, $\Omega_{a}$ is given by

$$
\Omega_{a}=\int_{a}^{b}\left(\sin x \sqrt{\frac{\sin ^{2} x \cos ^{2 n} x}{\left[H\left(\cos ^{n+1} x-\cos ^{n+1} a\right)+(\sin a) \cos ^{n} a\right]^{2}}-1}\right)^{-1} d x
$$

On the other hand, we note that a solution $s$ of (5.1) is invariant under the reflection,

$$
t_{0}+t \rightarrow t_{0}-t
$$

such that $s\left(t_{0}\right)=0$. Since reflections at $t=0, \Omega_{a}, 2 \Omega_{a}, 3 \Omega_{a}$, etc. act on $s, s$ is a periodic function on $R$ with period $\Omega_{a} . \gamma$ is a closed curve if and only if $\Omega_{a}$ is a rational multiple of $\pi$. Since $\Omega_{a}$ is a nonconstant, continuous function of $a$, we see that there exist countable closed curves for a given nonnegative number $H$. Since $\Re$ is parallel, so is $\mathcal{L}$. Collecting these results, we have

THEOREM 2. Let $N$ be an $n$-dimensional compact minimal submanifold in $S^{q}$. For a given nonnegative number $H$, there exist countable immersions of the compact manifold $S^{1} \times N$ into $S^{2+q}$ with parallel mean curvature vector with length $H$.

COROLlary 2. Theorem 2 holds for $S^{1} \times S^{q}$.

REMARK. When $H=0$, compact minimal hypersurfaces (except the Clifford torus which appeared in Corollary 2) are called Otsuki manifolds [7, 12].

In Theorem 2, if $N$ has the trivial normal connection, then (2) of Lemma 9 asserts that the normal connection of $S^{1} \times N$ is also flat. In the case that $N$ is a Clifford torus $T^{n-1}$ in $S^{2 n-3}$, we have 
COROllary 3. Let $H$ be a nonnegative number, then there exist countable immersions of $T^{n}$ into $S^{2 n-1}$ with trivial normal connection and parallel mean curvature vector with length $H$.

EXAMPLE 7. $\left(R_{+} \times R\right) \times{ }_{s} S^{q}$ is isometric to an open set of $R^{2+q}$. Let $N$ be an $n$-dimensional minimal submanifold and $\gamma$ a nonparametric curve $\gamma=(s(t), t)$ in $R_{+} \times R$ whose unit normal vector field $\mathcal{X}$ may be $(\partial / \partial s-\dot{s} \partial / \partial t) / \sqrt{1+\dot{s}^{2}}$. By a direct computation similar to Example 6, we obtain the ordinary differential equation of $s$ for a given nonnegative number $H$,

$$
\left(1 / \sqrt{1+\dot{s}^{2}}\right)^{2} \ddot{s}-n /\left(s \sqrt{1+\dot{s}^{2}}\right)= \pm(n+1) H .
$$

It implies that there is a constant $c$ such that

$$
\dot{s}^{2}=s^{2 n} /\left(c \mp((n+1) H / n) s^{n}\right)^{2}-1 .
$$

To guarantee $c+(n+1) H s^{n} / n>0$, we assume that the right-hand side of (5.5) is $-(n+1) H$ and $c>0$. Supposing that the initial conditions for $s$ satisfy

$$
s(0)=a, \quad \dot{s}(0)=0, \quad \ddot{s}(0)>0,
$$

we obtain $c=a^{n}(1-(n+1) H / n)$ and $a, 1<n /(n+1) H$. Since

$$
0<\lim _{s \rightarrow \infty} s^{2 n} /\left(c+((n+1) H / n) s^{n}\right)^{2}-1=(n /(n+1) H)^{2}-1<+\infty,
$$

$s$ is an increasing function on $[0, \infty)$. Using the symmetry $t \rightarrow-t$, we can get a positive function on $R$. Thus the length of a nonparametric curve $\gamma$ is $\infty$. Using $\gamma$ reparametrized by arc length $u$, we may regard $\gamma \times N$ as the warped product $R \times_{s(\gamma(u))} N$. From Lemma 1 , the completeness of $N$ shows that $\gamma \times N$ is complete. We obtain the following result analogous to Theorem 2 .

THEOREM 3. Let $N$ be an n-dimensional complete minimal submanifold in $S^{q}$ and $H$ a nonnegative number $<n /(n+1)$. Then there exists an immersion of $R \times N$ into $R^{2+q}$ such that the induced metric is complete and the mean curvature vector is parallel with length $H$.

REMARK. When $H=0$ and $N=S^{q}$ in Theorem 3, a minimal hypersurface $R \times S^{q}$ is a generalization of the catenoid [2].

Since every compact homogeneous space can be minimally immersed in $S^{n}$ [7], we get the following:

COROLlaRY 4. Let $N$ be a compact homogeneous space. Then $R \times N$ can be minimally immersed in $R^{n}$ as a complete submanifold.

EXAMPLE 8. $\left(R_{+} \times_{\cosh s} R\right) \times{ }_{\sinh s} S^{q}$ is isometric to an open set of $H^{2+q}$. Let $N$ be an $n$-dimensional minimal submanifold of $S^{q}$ and $\gamma$ a nonparametric curve $\gamma=$ $(s(t), t)$ in $R_{+} \times_{\cosh s} R$. Its unit normal vector field is

$$
((\cosh s) \partial / \partial s-\dot{s} /(\cosh s) \partial / \partial t) / \sqrt{\dot{s}^{2}+\cosh ^{2} s} \text {. }
$$

$\gamma \times N$ has constant mean curvature $H$ if and only if

$$
\begin{aligned}
\{(\cosh s)(\dot{s}-(\cosh s) & \left.(\sinh s))-2 \dot{s}^{2} \sinh s\right\} /{\sqrt{\dot{s}^{2}+\cosh ^{2} s}}^{3} \\
& -n \cosh ^{2} s /(\sinh s) \sqrt{\dot{s}^{2}+\cosh ^{2} s}= \pm(n+1) H
\end{aligned}
$$


Consequently, there is a constant $c$ such that

$$
\dot{s}^{2}=\cosh ^{2} s\left\{\left(\cosh ^{2} s\right)\left(\sinh ^{2 n} s\right) /\left[H \sinh ^{2} s \mp c\right]^{2}-1\right\} .
$$

We only consider the case that the right-hand side of $(5.7)$ is $-(n+1) H$ and $c>0$. Providing that the initial conditions of $s$ satisfy

$$
s(0)=a, \quad \dot{s}(0)=0, \quad \ddot{s}(0)>0,
$$

we see $c=(\cosh a) \sinh ^{n} a-H \sinh ^{n+1} a$ and

$$
\tanh a<1 / H, \quad \tanh ^{2} a-(n+1) H \tanh a+n>0 .
$$

For the sake of simplicity, we assume $1<\tanh a<1 / H$. This asserts the right-hand side of (5.8) has 0 only at $s=a$. Hence we must consider two cases:

$$
\text { There is } t_{0} \text { such that } t_{0}<\infty \text { and } \lim _{t \rightarrow t_{0}} s(t)=+\infty \text {, }
$$

$$
s \text { is an increasing function on }[0, \infty) \text {. }
$$

But the length of the curve is $\infty$ for any case. Thus $\gamma \times N$ is complete if so is $N$.

THEOREM 4. Let $N$ be an $n$-dimensional complete minimal submanifold in $S^{q}$. For a given nonnegative number $H<1$, there exist countable immersions of $R \times N$ into $H^{2+q}$ such that the induced metric is complete and the mean curvature vector is parallel with length $H$.

We obtain the same result as Corollary 4.

Corollary 5. Let $N$ be a compact homogeneous space. Then $R \times N$ can be minimally immersed in $\mathrm{H}^{n}$ such that the induced metric is complete.

REMARK. Using Examples 3 and 4, we obtain results similar to these theorems.

6. Second observation. Let $M$ be a $(2 n-1)$-dimensional Sasakian manifold with Sasakian structure $(\phi, \xi, \eta, g)$ and $N$ an $(n-1)$-dimensional integral submanifold in $M$ (i.e., $\xi$ is perpendicular to $N$ ). We denote by $\operatorname{Exp}_{x} t \xi$ a one parameter group of isometries generated by $\xi$. An orbit of $N$ is expressed in terms of a map $S$ of $R \times N$ into $M$ given by $S(t, x)=\operatorname{Exp}_{x} t \xi$. We observe that if $\operatorname{Exp}_{x} t \xi$ has a period (for example, $S^{2 n-1}$ has a period $2 \pi$ ), we may replace $R$ by $S^{1}$. The following is clear:

LEMMA 12. $S$ is an isometric immersion of the Riemannian product $R \times N$ into $M$. $\{t\} \times N$ for each $t \in R$ is also an integral submanifold whose second fundamental form $\sigma_{t}$ is expressed as $\sigma_{t}=\left(\operatorname{Exp}_{x} t \xi\right)_{*} \sigma$, where $\sigma$ is the second fundamental form of $N$.

Let $f$ and $h$ be functions on an interval $I_{1}$ with coordinate function $s$ such that $f>0$ and $f^{2}+h>0$. The same process as in $\S 3$ defines a metric $\tilde{g}$ on $I_{1} \times M$. A map $T$ of $I_{1} \times R \times N$ into $I_{1} \times M$ is naturally induced as $T(s, t, x)=\left(s, \operatorname{Exp}_{x} t \xi\right)$. It is clear that $T$ is an immersion with the induced metric $d s^{2}+\left(f^{2}+h\right) d t^{2}+f^{2} g$. We denote by $\sigma_{T}$ the second fundamental form of $T$. It follows from Lemma 5 that

$$
\begin{gathered}
\sigma_{T}(\partial / \partial s, \partial / \partial s)=\sigma_{T}(\partial / \partial s, \xi)=\sigma_{T}(\partial / \partial s, X)=\sigma_{T}(\xi, \xi)=0, \\
\sigma_{T}(\partial / \partial t, X)=\left(\left(f^{2}+h\right) / f^{2}\right) \phi X, \quad \sigma_{T}(X, Y)=\sigma_{t}(X, Y),
\end{gathered}
$$

where $X$ and $Y$ are vector fields on $N$. 
Let $I_{2}$ be an interval with coordinate function $u$ and $\gamma$ a regular curve $\gamma=$ $(s(u), t(u))$ of $I_{2}$ into $I_{1} \times R$. Then a map $\chi$ of $I_{2} \times N$ into $I_{1} \times M$ is defined by

$$
\chi(u, x)=\left(s(u), \operatorname{Exp}_{x} t(u) \xi\right) .
$$

Here we see that $\chi$ is an immersion with induced metric

$$
\left((\partial s / \partial u)^{2}+\left(f^{2}+h\right)(\partial t / \partial u)^{2}\right) d u^{2}+f^{2}(s(u)) g .
$$

Its second fundamental form is denoted by $\sigma_{\chi}$. Since $\chi$ is composed of $T$ and $U$ : $I_{2} \times N \rightarrow I_{1} \times R \times N$ such that $U(u, x)=(s(u), t(u), x)$, we obtain $\sigma_{x}=\sigma_{T}+\sigma_{U}$, where $\sigma_{U}$ is the second fundamental form of $U$. Again due to Lemma $5, \sigma_{U}$ satisfies

$$
\begin{gathered}
\sigma_{U}(\partial / \partial u, \partial / \partial u)=\sigma_{0}(\partial / \partial u, \partial / \partial u), \quad \sigma_{U}(\partial / \partial u, X)=0 \quad \text { and } \\
\sigma_{U}(X, Y)=-\sqrt{f^{2}+h} f f^{\prime}(\partial t / \partial u) / \sqrt{\left(f^{2}+h\right)(\partial t / \partial u)^{2}+(\partial s / \partial u)^{2}} g(X, Y) \Re,
\end{gathered}
$$

where $\sigma_{0}$ is the second fundamental form of $\gamma$ in $I_{1} \times R$ with metric

$$
d s^{2}+\left(f^{2}+h\right) d t^{2}
$$

and

$$
\Re=\frac{\left\{\sqrt{f^{2}+h}(\partial t / \partial u) \partial / \partial s-(\partial s / \partial u) / \sqrt{f^{2}+h} \partial / \partial t\right\}}{\sqrt{\left(f^{2}+h\right)(\partial t / \partial u)^{2}+(\partial s / \partial u)^{2}}} .
$$

Lemma 13. Let $X, Y$ be vector fields on $N$. Then

$$
\begin{gathered}
\sigma_{\chi}(\partial / \partial u, \partial / \partial u)=\sigma_{0}(\partial / \partial u, \partial / \partial u), \quad \sigma_{\chi}(\partial / \partial u, X)=\left(f^{2}+h\right) / f^{2} \phi X, \\
\sigma_{\chi}(X, Y)=\frac{-\sqrt{f^{2}+h} f f^{\prime}(\partial t / \partial u)}{\sqrt{\left(f^{2}+h\right)(\partial t / \partial u)^{2}+(\partial s / \partial u)^{2}}} g(X, Y) \Re+\sigma_{t}(X, Y) .
\end{gathered}
$$

Hence we get the mean curvature vector $\varrho$ of $I_{2} \times N$ in $I_{1} \times M$.

LEMMA 14.

$$
\begin{aligned}
\mathcal{E}=\left\{-(n-1) f^{\prime} \sqrt{f^{2}+h}\right. & (\partial t / \partial u) / \sqrt{\left(f^{2}+h\right)(\partial t / \partial u)^{2}+(\partial s / \partial u)^{2}} f \Re \\
& \left.+1 /\left(\dot{s}^{2}+\left(f^{2}+h\right)\right) \sigma_{0}(\partial / \partial u, \partial / \partial u)+(n-1) / f^{2} \mathcal{L}_{1}\right\} / n,
\end{aligned}
$$

where $\mathfrak{L}_{1}$ is the mean curvature vector of $N$.

We note that $E_{1}$ is perpendicular to the other parts of the right-hand side of Lemma 14, which, together with Lemma 11, implies the second observation.

THEOREM 5. $I_{2} \times N$ is minimal in $I_{1} \times M$ with metric $g$ if and only if

(1) $\gamma$ is a geodesic in $I_{1} \times R$ with metric

$$
f^{2(n-1)}\left(d s^{2}+\left(f^{2}+h\right) d t^{2}\right),
$$

(2) $N$ is minimal in $M$.

LEMMA 15. For the almost complex structure $J$ of $I_{1} \times M, I_{2} \times N$ is a totally real submanifold (i.e., $J T\left(I_{2} \times N\right)$ is the normal bundle of $I_{2} \times N$ in $\left.I_{1} \times M\right)$. 
7. Applications of the second observation. In this section, since we only consider $M=S^{2 n-1}$, we may assume that $\gamma$ is a curve in $I_{1} \times S^{1}$.

EXAMPLE 9. If $f=\sin s$ and $h=0$, then $(0, \pi) \times S^{2 n-1}$ with metric $\tilde{g}$ is the warped product $(0, \pi) \times{ }_{\sin s} S^{2 n-1}$ which is isometric to an open set of $S^{2 n}$. Let $N$ be an $(n-1)$-dimensional minimal integral submanifold in $S^{2 n-1}$ and $\gamma$ a nonparametric curve $(s(t), t)$ in $(0, \pi) \times_{\sin s} S^{1}$. According to Lemma 14, the mean curvature

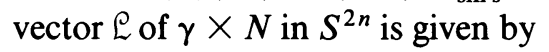

$$
\left\{1 /\left(\dot{s}^{2}+\sin ^{2} s\right) \sigma_{0}\left(\gamma_{*}, \gamma_{*}\right)-\left((n-1) \cos s / \sqrt{\dot{s}^{2}+\sin ^{2} s}\right) \Re\right\} / n,
$$

where $\gamma_{*}$ is the tangent vector of $\gamma$ and

$$
\Re=((\sin s) \partial / \partial s-(\dot{s} /(\sin s)) \partial / \partial t) / \sqrt{\dot{s}^{2}+\sin ^{2} s} .
$$

We see that the length of $\mathcal{L}$ is constant $H$ if and only if the innerproduct of $\Re$ and $\mathcal{L}$ is $H$ or $-H$, because $\mathcal{L}$ is proportional to $\Re$. On the other hand, $\sigma_{0}\left(\gamma_{*}, \gamma_{*}\right)$ is already calculated in Example 6. Thus we find the ordinary differential equation for $s$,

$$
\begin{aligned}
&\left\{(\sin s)(\ddot{s}-(\sin s)(\cos s))-2 \dot{s}^{2} \cos s\right\} /{\sqrt{\dot{s}^{2}+\sin ^{2} s}}^{3} \\
&-(n-1)(\cos s) / \sqrt{\dot{s}^{2}+\sin ^{2} s}= \pm H .
\end{aligned}
$$

Unfortunately, there is no first integral if $H \neq 0$. But if $H=0$, we have the first integral

$$
\left(\dot{s}^{2}+\sin ^{2} s\right) / \sin ^{2(n+1)} s,
$$

which implies that there exists a positive constant $c$ such that

$$
\dot{s}^{2}=\sin ^{2} s\left(c \sin ^{2 n} s-1\right) .
$$

Assume that $s$ satisfies the initial conditions such that

$$
s(0)=a, \quad \dot{s}(0)=0, \quad \ddot{s}(0) \geqslant 0 .
$$

Using (7.1) and (7.2), we obtain $c=\sin ^{n} a$ and $0<a \leqslant \pi / 2$. It is understood that $a=\pi / 2$ gives a constant solution $s=\pi / 2$ which corresponds to a minimal submanifold

$$
S: S^{1} \times N \rightarrow S^{2 n-1} \subset S^{2 n} .
$$

Hence we may assume $a<\pi / 2$. Using procedures discussed in Example 6, $s$ is a periodic function on $R$ with period

$$
\Omega_{a}=\int_{a}^{a+\pi / 2}\left(\sin x \sqrt{\frac{\sin ^{2 n} x}{\sin ^{2 n} a}-1}\right)^{-1} d x
$$

Note that the curve $s=s(t)$ is closed in $(0, \pi) \times_{\sin s} S^{1}$ if and only if the period $\Omega_{a}$ is a rational multiple of $\pi$. Hence there exist countable closed curves.

THEOREM 6. Let $N$ be an $(n-1)$-dimensional compact minimal integral submanifold in $S^{2 n-1}$. Then there exist countable minimal immersions of the compact manifold $S^{1} \times N$ into $S^{2 n}$, which are not contained in a totally geodesic hypersphere of $S^{2 n}$ except example (7.3). 
COROLlARY 6. Theorem 6 holds for $N=S^{n-1}$.

In particular, if $n=2$, we have countable minimal immersions of $T^{2}$ into $S^{4}$. On the other hand, H. B. Lawson, Jr. [8] constructs minimal immersions of $T^{2}$ into $S^{4}$, which are called bipolar surfaces, by a different method. It seems that our examples are contained in them.

Since K. Yano and M. Kon give an example of a minimal immersion of a flat torus $T^{n-1}$ into $S^{2 n-1}$ such that $T^{n-1}$ is an integral submanifold [16, p. 133], we get

COROLlaRY 7. There exist countable minimal immersions of $T^{n}$ into $S^{2 n}$ which are not contained in a totally geodesic hypersphere of $S^{2 n}$ except case (7.3).

ExAmple 10. From Lemma $8,(0, \pi / 2) \times S^{2 n-1}$ with metric $d s^{2}+\sin ^{2} s g+$ $\sin ^{2} s\left(\cos ^{2} s-1\right) \eta \otimes \eta$ is isometric to a geodesic coordinate neighborhood of $P_{n}$. Let $N$ be an $(n-1)$-dimensional minimal integral submanifold in $S^{2 n-1}$ and $\gamma$ a nonparametric curve $\gamma(t)=(s(t), t)$ in $(0, \pi / 2) \times S^{1}$ with metric $d s^{2}+$ $\sin ^{2} s \cos ^{2} s d t^{2}$. By Lemma 14, the mean curvature vector $\mathcal{L}$ of $\gamma \times N$ in $P_{n}$ is given by

$$
\left\{1 /\left(\dot{s}^{2}+\sin ^{2} s\right) \sigma_{0}\left(\gamma_{*}, \gamma_{*}\right)-(n-1) \cos ^{2} s / \sqrt{\dot{s}^{2}+\sin ^{2} s} \Re\right\} / n
$$

where $\sigma_{0}$ is the second fundamental form of $\gamma$ in $(0, \pi / 2) \times S^{1}$ with metric $d s^{2}+\sin ^{2} s \cos ^{2} s d t^{2}$ and $\mathscr{T}$ is the unit normal vector field of $\gamma$ such that

$$
\Re=\{(\sin s)(\cos s) \partial / \partial s-(\dot{s} /(\sin s)(\cos s)) \partial / \partial t\} / \sqrt{\dot{s}^{2}+\sin ^{2} s \cos ^{2} s} .
$$

Consequently the condition that $\mathcal{L}$ has a constant length $H$ is equivalent to

$$
\begin{gathered}
\left\{((\sin s)(\cos s)) \ddot{s}-\left(\cos ^{2} s-\sin ^{2} s\right)\left(\sin ^{2} s \cos ^{2} s+2 \dot{s}^{2}\right)\right\} /{\sqrt{\dot{s}^{2}+\sin ^{2} s \cos ^{2} s}}^{3} \\
-(n-1) \cos ^{2} s / \sqrt{\dot{s}^{2}+\sin ^{2} s \cos ^{2} s}= \pm n H .
\end{gathered}
$$

This implies that there exists a constant $c$ such that

$$
\dot{s}^{2}=\sin ^{2} s \cos ^{2} s\left\{\sin ^{2 n} s \cos ^{2} s /[c \mp(n H /(n+1))]^{2}-1\right\} .
$$

In order to simplify the analysis of solutions of (7.4), we assume that the right-hand side of (7.4) is $n H$ and $c>0$. Now we choose initial conditions of $s$ as

$$
s(0)=a, \quad \dot{s}(0)=0, \quad \ddot{s}(0)>0 .
$$

Under these initial conditions, we can apply the same argument as Example 6 to see that $s$ is a periodic function on $R$ with period

$$
\Omega_{a}=\int_{a}^{b} \frac{d x}{(\sin x)(\cos x) \sqrt{\frac{\sin ^{2 n} x \cos ^{2} x}{\left[n H\left(\sin ^{n+1} x-\sin ^{n+1} a\right) /(n+1)+\sin ^{n} a \cos a\right]^{2}}}-1},
$$

where $a$ and $b$ satisfy

$$
\tan a<\min \left[\left\{-n H+\sqrt{(n H)^{2}+4 n}\right\} / 2,(n+1) / n H\right]
$$


and

$$
\sin ^{n} b\{\cos b-n H(\sin b) /(n+1)\}=\sin ^{n} s\{\cos a-n H(\sin a) /(n+1)\} .
$$

Hence there exist countable closed curves for each $H$. These, together with Lemma 15 , assert

THEOREM 7. Let $N$ be an $(n-1)$-dimensional compact minimal integral submanifold in $S^{2 n-1}$ and $H$ a nonnegative number. Then there exist countably totally real immersions of the compact manifold $S^{1} \times N$ into $P_{n}$ with constant mean curvature $H$.

COROLlaRY 8. Theorem 7 holds for $S^{1} \times S^{n-1}$.

We also obtain a result similar to Corollary 7.

COROllary 9. Let $H$ be a nonnegative number. Then there exist countable totally real immersions of $T^{n}$ into $P_{n}$ with constant mean curvature $H$.

REMARK. (1) If $H=0$ in Corollary 8 , then the constant solution of (7.4) corresponds to a Riemannian product $S^{1}((\sin a)(\cos a)) \times S^{n-1}(\sin a)$, where $S^{l}(r)$ is an $l$-dimensional sphere with radius $r$ and $\tan a=\sqrt{n}$. These examples are treated in [6] (if $n=2$ ) and [9] (a general case).

(2) Needless to say, we can construct totally real submanifolds with constant mean curvature in $\mathrm{C}^{n}$ and $H_{n}$.

8. The relation between some pinching theorems and examples. First we state some pinching theorems.

Proposition 1 [10]. Let $M$ be a connected compact Riemannian manifold of dimension $n$ with nonnegative sectional curvature. If $\psi: M \rightarrow S^{n+1}$ is an isometric immersion with constant mean curvature, then

(1) $\psi(M)$ is a great or small sphere of $S^{n+1}$, and $\psi$ is an imbedding,

(2) $\psi(M)$ is a product of spheres $S^{p}\left(r_{1}\right) \times S^{q}\left(r_{2}\right)\left(r_{1}^{2}+r_{2}^{2}=1\right)$ and $p \neq 1, n-1, \psi$ is an imbedding.

Proposition $2[5,15]$. Let $M$ be an $n$-dimensional complete submanifold of nonnegative sectional curvature in $R^{m}$ or $S^{m}$. Suppose that the mean curvature vector is parallel in the normal bundle and the normal connection is flat. If $M$ is either compact or has constant scalar curvature, then $M$ is the product manifold $S^{n} 1\left(r_{1}\right) \times \cdots \times S^{n} k\left(r_{k}\right)$.

Proposition $3[3,17]$. Let $M$ be a compact totally real minimal surface in $P_{2}$. If $M$ has nonnegative sectional curvature, then $M$ is totally geodesic or flat.

Proposition 4 [4]. Let $M$ be a 4-dimensional compact orientable conformally flat totally real minimal submanifold in $P_{4}$ with nonnegative Euler number. If $M$ has the scalar curvature $\rho$ between 0 and $15 / 2$, then $\rho=0$, or $15 / 2$.

It is easily seen that the examples for Corollaries 2, 3, $8(n=2), 8(n=4)$ and 9 $(n=4)$, respectively, correspond to Propositions 1,2, 3 and 4 and examples for each constant solution attain each pinching value. Considering the initial conditions near that of constant solutions, we, roughly speaking, know that the relaxation of pinching values is impossible. 


\section{REFERENCES}

1. R. L. Bishop and B. O’Neill, Manifolds of negative curvature, Trans. Amer. Math. Soc. 145 (1969), $1-49$.

2. D. Blair, $A$ generalization of the catenoid, Canad. J. Math. 27 (1975), 231-236.

3. B. Y. Chen and K. Ogiue, On totally real submanifolds, Trans. Amer. Math. Soc. 193 (1973), 257-266.

4. N. Ejiri, Totally real minimal submanifolds in a complex projective space, preprint.

5. J. A. Erbacher, Isometric immersions with constant mean curvature and triviality of the normal bundle, Nagoya Math. J. 45 (1972), 139-165.

6. C. S. Houh, Some totally real minimal surface in $C P^{2}$, Proc. Amer. Math. Soc. 40 (1973), 240-244.

7. W. Y. Hsiang and H. B. Lawson, Jr., Minimal submanifolds of low-cohomogeneity, J. Differential Geom. 5 (1971), 1-38.

8. H. B. Lawson, Jr., Complete minimal surfaces in $S^{3}$, Ann. of Math. (2) 90 (1970), 335-374.

9. H. Naitoh, Totally real parallel submanifolds in $P^{n}(C)$, Tokyo J. Math. 4 (1981), 279-306.

10. K. Nomizu and B. Smyth, $A$ formula of Simon's type and hypersurfaces with constant mean curvature, J. Differential Geom. 3 (1969), 367-377.

11. K. Ogiue, On fiberings of almost contact manifolds, Kōdai Math. Sem. Rep. 17 (1965), 53-62.

12. T. Otsuki, Minimal hypersurfaces in a Riemannian manifold of constant curvature, Amer. J. Math. 92 (1970), 145-173.

13. J. Simons, Minimal varieties in Riemannian manifolds, Ann. of Math. (2) 88 (1968), 62-105.

14. S. Tanno, Sasakian manifolds with constant $\phi$-holomorphic sectional curvature, Tôhoku Math. J. 21 (1969), 501-507.

15. K. Yano and S. Ishihara, Submanifolds with parallel mean curvature vector, J. Differential Geom. 6 (1971), 95-118.

16. K. Yano and M. Kon, Anti-invariant submanifolds, Marcel Dekker, New York, 1976.

17. S. T. Yau, Submanifolds with constant mean curvature. I, Amer. J. Math. 97 (1975), 76-100.

Department of Mathematics, Tokyo Metropolitan University, Tokyo 158, JaPan 